\title{
Occurrence of complete atrioventricular block during surgery
}

\author{
Jong-Min Byun, Chul-Woo Jung, and Seung-Yeon Shin \\ Department of Anesthesiology and Pain Medicine, Seoul National University Hospital, Seoul, Korea
}

Complete atrioventricular block (AVB) in the heart results in serious hemodynamic instability that is often refractory to vagolytic medication; therefore, pacemaker insertion prior to surgery is mandatory [1]. Conversely, first degree AVB, a benign cardiac dysrhythmia, does not have definite cardiovascular symptoms and therefore does not require preoperative treatment. Furthermore, conversion from first degree AVB to complete AVB is rare. Herein, we report a case of sudden onset of complete AVB in a patient with first degree AVB that occurred preoperatively and provide a literature review.

An 83-year-old woman $(49 \mathrm{~kg} / 144 \mathrm{~cm}$, ASA physical status II) was admitted to receive an incisional hernia repair. She had a previous history of two operations under general anesthesia without event. Routine preoperative tests showed no abnormal findings except for first degree AVB on electrocardiogram (ECG). Upon arrival in the operating room without premedication, standard monitoring of ECG, pulse oximetry, and noninvasive blood pressure were established. ECG revealed a first degree AVB pattern similar to preoperative (Fig. 1A). Anesthesia was induced with propofol and rocuronium and maintained with sevoflurane-air in oxygen. Intraoperative vital signs were stable (Fig. 1B), except that the heart rate decreased to 35 beats/min two hours after initiation of the operation (Fig. 1C), which was recovered to 52 beats/min after administration of $0.5 \mathrm{mg}$ atropine intravenously. At the end of the operation, $1.0 \mathrm{mg}$ atropine and $1.5 \mathrm{mg}$ neostigmine were administered for reversal of muscle relaxation after confirming that the exhaled gas concentration was low and vital signs were stable (Fig. 1D). Within a few minutes, the heart rate decreased abruptly to 31 beats/min with apparent ECG change (Fig. 1E). Intravenous atropine $(0.5 \mathrm{mg})$ was administered immediately, but severe bradycardia persisted. Because the bradycardia was refractory to vagolytic medication, we inserted a temporary pacemaker. The radial arterial line was placed instantly for continuous arterial pressure monitoring and blood sampling. Arterial blood gas, electrolytes and cardiac enzymes showed no remarkable findings. Owing to equipment and man power, the patient was transferred to the cardiac intervention room. Midazolam (1.5 mg) was administered to secure sedation during transport. While transferring to the intervention room, a sudden fall in systolic arterial pressure to $60 \mathrm{mmHg}$ was observed. Hypotension was recovered after two intravenous administrations of epinephrine $100 \mathrm{mcg}$, but bradycardia continued (Fig. 1F). At the cardiac intervention room, a temporary pacemaker was successfully inserted and set in VVI mode with a rate of 60 beats $/ \mathrm{min}$. Echocardiogram demonstrated normal ventricular contractibility without regional wall motion abnormality. Cardiac enzyme concentrations showed mild elevation of troponin I at six hours postoperatively. Continuous infusion of low molecular weight heparin and nitroglycerine were started. The plasma concentration of troponin I decreased to normal on post-operative day (POD) 4 . As the patient was alert without cardiac symptoms, the temporary pacemaker was turned off transiently under close observation on POD 4. Complete AVB was no longer found on ECG over 24 hours; therefore, the pacemaker was removed from the patient on POD 6. Coronary CT on POD 8 revealed mild atherosclerotic change without focal stenotic lesion. The patient was discharged

Corresponding author: Chul-Woo Jung, M.D., Department of Anesthesiology and Pain Medicine, Seoul National University Hospital, 101 Daehang-no, Jongno-gu, Seoul 110-744, Korea. Tel: 82-2-2072-0640, Fax: 82-2-747-5639, E-mail: spss@snuh.org

(c) This is an open-access article distributed under the terms of the Creative Commons Attribution Non-Commercial License (http:// creativecommons.org/licenses/by-nc/3.0/), which permits unrestricted non-commercial use, distribution, and reproduction in any medium, provided the original work is properly cited. 


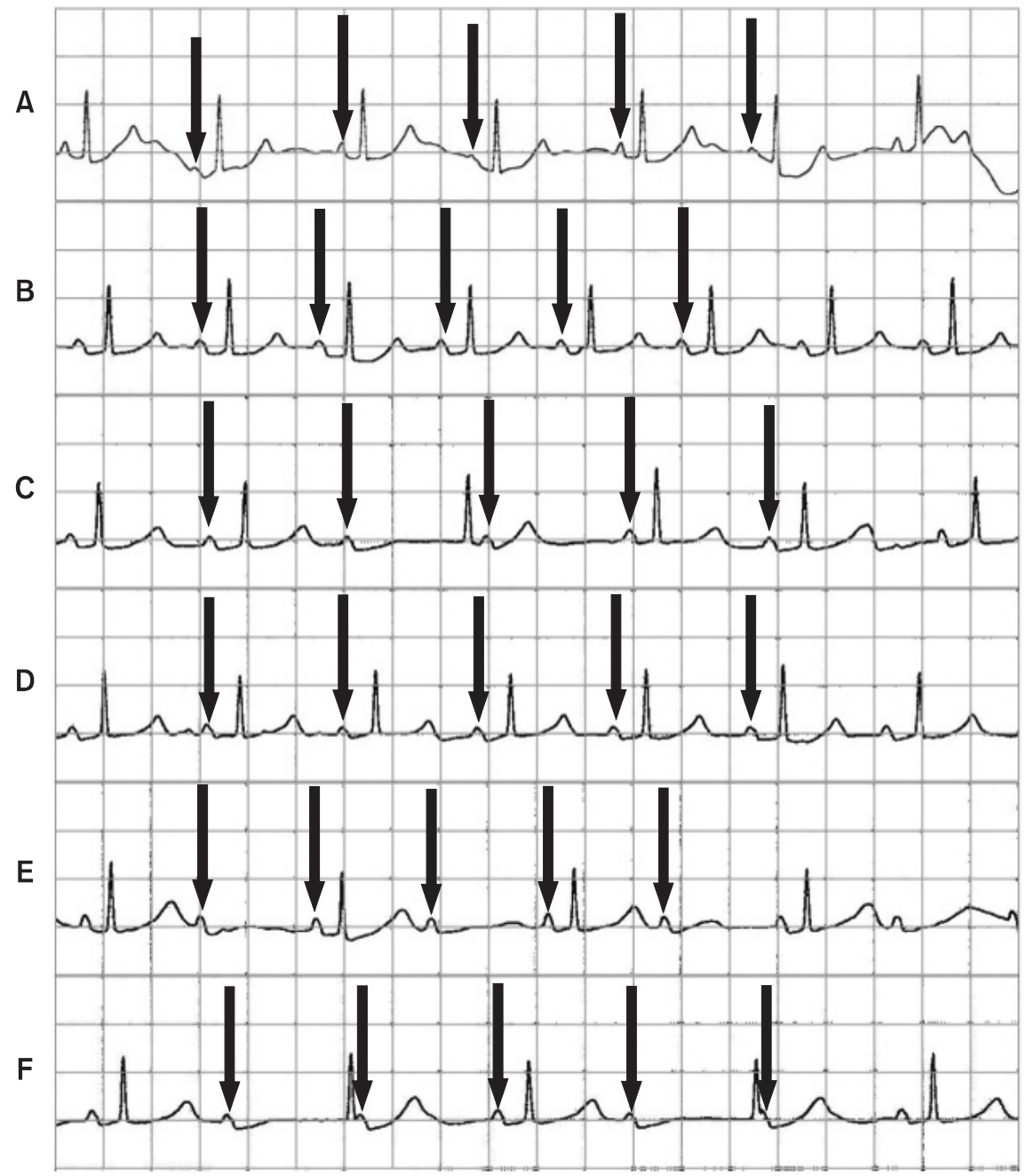

\title{
Before anesthesia induction \\ $\mathrm{HR}=52$ \\ SAP/DAP/MAP $=154 / 82 / 115$

\author{
Intraoperative 1 \\ $\mathrm{HR}=60$
} \\ SAP/DAP/MAP $=141 / 75 / 95$
}

\author{
Intraoperative 2 \\ $\mathrm{HR}=35$ \\ SAP/DAP/MAP $=109 / 63 / 76$
}

\author{
At the end of surgery \\ $\mathrm{HR}=53$ \\ SAP/DAP/MAP $=115 / 74 / 97$
}
After injection of atopine and neostigmine $\mathrm{HR}=31$
SAP/DAP/MAP = 148/81/104

Before transport to cardiac intervention room $\mathrm{HR}=35$

SAP/DAP/MAP $=132 / 58 / 85$

Fig. 1. Changes in electrocardiogram and vital signs during operation. Electrocardiogram waveforms and vital signs were retrieved from the data server of electric medical recording system postoperatively. The arrows indicate regular P-waves. HR: heart rate, SAP: systolic arterial pressure, DAP: diastolic arterial pressure, MAP: mean arterial pressure.

without any cardiovascular symptoms on POD 12. Two months later, follow-up ECG revealed sinus bradycardia ( 56 beats/min) with first degree AVB, which was similar to the preoperative findings.

In acquired complete $\mathrm{AVB}$, the site of the block resides in the His-Purkinje system distal to the atrioventricular (AV) node. In acquired type, the ECG primarily shows a wide QRS complex and relatively slow heart rate [2]. However, our patient showed normal QRS and severe bradycardia that was refractory to atropine medication. This rare and atypical AVB is referred to as paroxysmal atrioventricular block [3]. The precise mechanism of paroxysmal AVB remains unclear, but is thought to be related to the hyper reactivity of the AV node to vagotonic stimulation. Medications such as digitalis and antiarrhythmic drugs, coronary artery disease, and aging-related degenerative changes in the conduction system are common causes of paroxysmal
AVB among various causes, including surgery, electrolyte imbalance, endocarditis, tumor, Chagas' disease, rheumatic heart disease, calcified aortic valve stenosis, myxedema and inflammatory and infiltrative heart diseases. The possible mechanism in this case may be assumed to be preexisting degenerative change in the conduction system. This is inferred from the presence of a first degree AV block in an elderly patient, which might be triggered by vagotonic stimuli such as surgical manipulation of the hernia sac and administration of neostigmine. A similar case was reported by Lee et al. [4], who found that an asymptomatic female developed high grade heart block during cesarean section under general anesthesia with enflurane. Postoperative ECG revealed that she had underlying Mobitz type I second degree AVB. The authors assumed that pre-existing heart block might be a risk factor for development of high grade AVB and the progression of the block might be 
associated with vagal reflex related to uterine traction. Finally, we point out that the onset of complete AVB (Fig. 1C) was not noticed by the attending anesthesiologist until neostigmine was administered (Fig. 1E). Lack of vigilance was the primary cause of late detection of complete AVB. However, lack of a freeze option in the ECG panel of the monitor system (Solar ${ }^{\circledR}$ $8000 \mathrm{M}$ patient monitor, GE Healthcare, WI) might have been another cause of delayed diagnosis. This is because we only managed to conclude that complete AVB had already occurred during operation after reviewing the ECG waveforms that were retrieved from the data storage server postoperatively.

In conclusion, we report here a case of sudden onset of complete AVB in an elderly patient with first degree AVB that occurred preoperatively. During anesthetic management of such patients, vigilant monitoring of cardiac rhythms is mandatory. Preparation of a temporary pacemaker is also recommended because such patients might be refractory to vagolytic medications.

\section{References}

1. Epstein AE, DiMarco JP, Ellenbogen KA, Estes NA 3rd, Freedman RA, Gettes LS, et al. ACC/AHA/HRS 2008 Guidelines for DeviceBased Therapy of Cardiac Rhythm Abnormalities: a report of the American College of Cardiology/American Heart Association Task Force on Practice Guidelines (Writing Committee to Revise the ACC/AHA/NASPE 2002 Guideline Update for Implantation of Cardiac Pacemakers and Antiarrhythmia Devices): developed in collaboration with the American Association for Thoracic Surgery and Society of Thoracic Surgeons. Circulation 2008; 117: e350-408.

2. Zipes DP. Special arrhythmias: diagnosis and treatment. In: Heart disease. 5th ed. Edited by Braunwald E: Philadelphia, S.B.Saunders Company. 1997, pp 687-93.

3. Shohat-Zabarski R, Iakobishvili Z, Kusniec J, Mazur A, Strasberg B. Paroxysmal atrioventricular block: clinical experience with 20 patients. Int J Cardiol 2004; 97: 399-405.

4. Lee HJ, Chon JY, Oh YS, Kim NH. High Grade Atrioventricular Block during Cesarean Section under General Anesthesia in a Parturient with Mobitz Type I Atrioventricular Block. Korean J Anesthesiol 2006; 51: 641-3. 